\title{
Nonsequential double ionization of helium in IR+XUV two-color laser fields II: Collision-excitation ionization process
}

\author{
Facheng Jin ${ }^{1,2}$, Jing Chen ${ }^{3}$, Yujun Yang ${ }^{4}$, Xiaojun \\ Liu $^{5}$, Zong-Chao Yan ${ }^{6}$, and Bingbing Wang1* \\ ${ }^{1}$ Laboratory of Optical Physics, Beijing National Laboratory for Condensed Matter Physics, \\ Institute of Physics, Chinese Academy of Sciences, Beijing 100190, China \\ ${ }^{2}$ College of Science, Xi'an Aeronautical University, Xi'an 71007\%, China \\ ${ }^{3}$ Institute of Applied Physics and Computational Mathematics, \\ P. O. Box 8009, Beijing 100088, China \\ ${ }^{4}$ Institute of Atomic and Molecular Physics, \\ Jilin University, Changchun 130012, China \\ ${ }^{5}$ State Key Laboratory of Magnetic Resonance and Atomic and Molecular Physics, \\ Wuhan Institute of Physics and Mathematics, \\ Chinese Academy of Sciences, Wuhan 430071, China and \\ ${ }^{6}$ Department of Physics, University of New Brunswick, \\ Fredericton, New Brunswick, Canada E3B 5 A3
}

(Dated: September 18, 2018) 


\begin{abstract}
The collision-ionization mechanism of nonsequential double ionization (NSDI) process in IR+XUV two-color laser fields [Phys. Rev. A 93, 043417 (2016)] has been investigated by us recently. Here we extend this work to study the collision-excitation-ionization (CEI) mechanism of NSDI processes in the two-color laser fields with different laser conditions. It is found that the CEI mechanism makes a dominant contribution to the NSDI as the XUV photon energy is smaller than the ionization threshold of the $\mathrm{He}^{+}$ion, and the momentum spectrum shows complex interference patterns and symmetrical structures. By channel analysis, we find that, as the energy carried by the recollision electron is not enough to excite the bound electron, the bound electron will absorb XUV photons during their collision; as a result, both forward and backward collisions make a comparable contributions to the NSDI processes. However, it is found that, as the energy carried by the recollision electron is large enough to excite the bound electron, the bound electron does not absorb any XUV photon and it is excited only by sharing the energy carried by the recollsion electron, hence the forward collision plays a dominant role on the NSDI processes. Moreover, we find that the interference patterns of the NSDI spectra can be reconstructed by the spectra of two above-threshold ionization (ATI) processes, which may be used to analyze the structure of the two separate ATI spectra by NSDI processes.
\end{abstract}

${ }^{*}$ Electronic address: 
*wbb@aphy.iphy.ac.cn

\section{INTRODUCTION}

Nonsequential double ionization (NSDI) of atoms and molecules in a strong infrared (IR) laser field has continued to attract considerable interest [1] since the first observation of the knee structure in the doubly charged ion yield curve as a function of laser intensity for Xe atom [2]. Up to now, it is widely accepted that the recollision picture is responsible for the NSDI [3]. In this picture, one electron is released via a tunneling process, then accelerated, and finally driven back to collide with another electron making two electrons simultaneously ionized by the IR laser field, which is called the collision-ionization (CI) process; whereas if the second electron is pumped to an excited state, and then ionized subsequently by the IR laser field, this process is called the collision-excitation-ionization (CEI). With great advances in the study of the NSDI by many theoretical approaches [4-17], for example, solving the full-dimensional time-dependent Schrödinger equation [4], the S-matrix theory [5-9], semiclassical [10-12] and classical [13, 14] models as well as the frequency-domain theory [15-17], both the CI and CEI mechanisms have been investigated in the NSDI process. Especially in recent years, more attention has been paid to the CEI mechanism of NSDI because it involves more complex electron correlation dynamics and may give rise to nontrivial NSDI features. Indeed, a number of interesting NSDI phenomena have been observed recently [9, 18 26], such as the cross-shaped in the correlated electron momentum distribution from NSDI with a near-single-cycle [18, 19], the dependence of momentum distribution on laser intensities and carrier-envelope phases [21, 22], and quantum and depletion effects below the threshold intensity [9], as well as the nonstructured momentum distribution of high-Z rare-gas atoms [25]. These results are believed to be closely related to the CEI mechanism and further stimulate the study of NSDI process caused by CEI.

With rapid development of free-electron laser technology [27-29] and application of the high-order harmonic generation [30 32], NSDI can take place in an extreme ultraviolet (XUV) laser field. Applying XUV light source, a great progress has been made in understanding the NSDI dynamics in this short wavelength regime [33, 34]. For example, the virtual sequential model [35] and the joint angular distribution [36, 37] show the effect of the electron correlation in the NSDI process. More recently, NSDI dynamics has been further 
explored in the combined IR and XUV fields. Hu [38] first calculated the double ionization of helium in IR+XUV two-color laser fields by ab initio calculation. It was found that the electron correlation can significantly enhance the NSDI probability by controlling the XUV laser field to excite the bound electron of $\mathrm{He}^{+}$ion. Liu et al. [39] investigated the dependence of joint photoelectron angular distributions on the energy sharing of two ionized electrons by solving the time-dependent Schrödinger equation in full dimensionality. It was found that the joint photoelectron angular distribution in IR+XUV two-color laser fields is different from that in a monochromatic XUV laser field.

With the help of formal scattering theory [40], the frequency-domain theory based on the nonperturbative quantum electrodynamics was developed by Guo et al. [41]. This approach has been successfully extended to deal with the recollision processes, such as the high-order above-threshold ionization (HATI) [42, 43], high-order harmonic generation (HHG) [44, 45] and the NSDI [15] in a monochromatic IR laser field. One major advantage of this frequencydomain theory is that all of the recollision processes can be decoupled into certain channels, which can be further investigated separately. Also, the approach can save a large amount of computation time because of its nature of time-independence. Recently, the frequencydomain theory has been employed to investigate the above-threshold ionization (ATI) in IR+XUV two-color laser fields [46, 47]. Furthermore, we have studied the momentum spectrum of two ionized electrons and analyzed the formation of momentum spectrum in the NSDI due to the CI mechanism in the two-color laser fields [16, 17]. In this paper, we shall extend the frequency-domain theory to investigate the CEI mechanism of NSDI in IR+XUV two-color laser fields. By the channel analysis, we investigate the channel contributions to the momentum distribution of two ionized electrons as well as the contributions of the forward and backward collisions on NSDI. Moreover, by employing the saddle-point approximation, we obtain the energy conservation equations satisfied by the two ionized electrons and illustrate the formation of the momentum spectrum.

This paper is organized as follows. Section II introduces the theoretical method. In Sec. III, we present the numerical results and a comprehensive analysis of them. Finally, in Sec. IV our conclusions are given. Atomic units are used throughout unless otherwise stated. 


\section{THEORETICAL METHOD}

The Hamiltonian for the system of a two-electron atom in two-color linearly polarized laser fields with frequencies of $\omega_{1}$ and $\omega_{2}$ is [41]

$$
H=H_{0}+U+V
$$

where

$$
H_{0}=\frac{\left(-i \nabla_{1}\right)^{2}}{2}+\frac{\left(-i \nabla_{2}\right)^{2}}{2}+\omega_{1} N_{1}+\omega_{2} N_{2}
$$

is the free electron and photon energy operator for the laser-matter system. $N_{i}$ is the photon number operator for laser field with frequency $\omega_{i}$ for $i=1,2$. The Coulomb potential is $U=U_{1}+U_{2}+U_{12}$, where $U_{i}$ for $i=1,2$ is the interaction potential between electron and nuclei, and $U_{12}$ is the interaction potential between the two electrons. Finally, the electron-photon interaction potential is $V=V_{1}+V_{2}$, where

$$
\begin{aligned}
V_{j}= & -\left[\left(-i \nabla_{j}\right) \cdot \mathbf{A}_{1}\left(-\mathbf{k}_{1} \cdot \mathbf{r}_{j}\right)+\left(-i \nabla_{j}\right) \cdot \mathbf{A}_{2}\left(-\mathbf{k}_{2} \cdot \mathbf{r}_{j}\right)\right] \\
& +\frac{1}{2}\left[\mathbf{A}_{1}\left(-\mathbf{k}_{1} \cdot \mathbf{r}_{j}\right)+\mathbf{A}_{2}\left(-\mathbf{k}_{2} \cdot \mathbf{r}_{j}\right)\right]^{2},
\end{aligned}
$$

$\mathbf{A}_{s}\left(-\mathbf{k}_{s} \cdot \mathbf{r}_{j}\right)=\left(2 V_{s} \omega_{s}\right)^{-1 / 2}\left(\hat{\varepsilon}_{s} e^{i \mathbf{k}_{s} \cdot \mathbf{r}_{j}} a_{s}+c . c\right.$. $)$ is the vector potential with $j=1,2, \mathbf{k}_{s}$ is the wave vector, $\hat{\varepsilon}_{s}$ is the polarization vector, $a_{s}\left(a_{s}^{\dagger}\right)$ is the annihilation (creation) operator, and $V_{s}$ is the normalization volume of the laser field with the frequency $\omega_{s}$ for $s=1,2$.

The transition matrix from the initial state $\left|\Psi_{i}\right\rangle$ to the final state $\left|\Psi_{f}\right\rangle$ for a NSDI process caused by the CEI mechanism is [15]

$$
T_{\mathrm{CEI}}=\left\langle\Psi_{f}\left|V_{2} \frac{1}{E_{f}-H-i \varepsilon} U_{12} \frac{1}{E_{i}-H+i \varepsilon} V_{1}\right| \Psi_{i}\right\rangle .
$$

In the above, the initial state for the system is $\left|\Psi_{i}\right\rangle=\left|\Psi_{i n_{1} n_{2}}\left(\mathbf{r}_{1}, \mathbf{r}_{2}\right)\right\rangle=\left|\Phi_{1}\left(\mathbf{r}_{1}, \mathbf{r}_{2}\right)\right\rangle \otimes$ $\left|n_{1}\right\rangle \otimes\left|n_{2}\right\rangle$, where $\left|\Phi_{1}\left(\mathbf{r}_{1}, \mathbf{r}_{2}\right)\right\rangle$ is the ground state of the atom, and $\left|n_{j}\right\rangle$ is the Fock state of the laser field with the photon number $n_{j}$ for $j=1,2$. The initial energy of the system is $E_{i}=-\left(I_{p_{1}}+I_{p_{2}}\right)+\left(n_{1}+1 / 2\right) \omega_{1}+\left(n_{2}+1 / 2\right) \omega_{2}$ with $I_{p_{1}}\left(I_{p_{2}}\right)$ being the first (second) ionization potential of the atom. The final state $\left|\Psi_{f}\right\rangle=\left|\Psi_{\mathbf{p}_{1} \mathbf{p}_{2} k_{1} k_{2}}\left(\mathbf{r}_{1}, \mathbf{r}_{2}\right)\right\rangle$ is the quantized-field Volkov state of two electrons in a two-color laser field [15, 41]

$$
\begin{aligned}
& \left|\Psi_{\mathbf{p}_{1} \mathbf{p}_{2} k_{1} k_{2}}\left(\mathbf{r}_{1}, \mathbf{r}_{2}\right)\right\rangle=V_{e}^{-1} \exp \left\{i\left[\left(\mathbf{p}_{1}+u_{p_{1}} \mathbf{k}_{1}+u_{p_{2}} \mathbf{k}_{2}\right) \cdot \mathbf{r}_{1}+\left(\mathbf{p}_{2}+u_{p_{1}} \mathbf{k}_{1}+u_{p_{2}} \mathbf{k}_{2}\right) \cdot \mathbf{r}_{2}\right]\right\} \\
& \quad \times \sum_{j_{1}=-k_{1}, j_{2}=-k_{2}}^{\infty} \aleph_{j_{1} j_{2}}\left(\zeta_{1}\right)^{*} \exp \left\{-i\left[j_{1}\left(\mathbf{k}_{1} \cdot \mathbf{r}_{1}+\phi_{1}\right)+j_{2}\left(\mathbf{k}_{2} \cdot \mathbf{r}_{1}+\phi_{2}\right)\right]\right\} \\
& \quad \times \sum_{j_{3}=-k_{1}-j_{1}, j_{4}=-k_{2}-j_{2}}^{\infty} \aleph_{j_{3} j_{4}}\left(\zeta_{2}\right)^{*} \exp \left\{-i\left[j_{3}\left(\mathbf{k}_{1} \cdot \mathbf{r}_{2}+\phi_{1}\right)+j_{4}\left(\mathbf{k}_{2} \cdot \mathbf{r}_{2}+\phi_{2}\right)\right]\right\} \\
& \quad \times\left|k_{1}+j_{1}+j_{3}, k_{2}+j_{2}+j_{4}\right\rangle,
\end{aligned}
$$


with the energy $E_{f}=\mathbf{p}_{1}{ }^{2} / 2+\mathbf{p}_{2}{ }^{2} / 2+\left(k_{1}+1 / 2\right) \omega_{1}+\left(k_{2}+1 / 2\right) \omega_{2}+2 u_{p_{1}} \omega_{1}+2 u_{p_{2}} \omega_{2}$, where $u_{p_{j}}$ and $\phi_{j}$ are the ponderomotive energy in units of frequency $\omega_{j}$ and the initial phase of the laser field with frequency $\omega_{j}$ for $j=1,2$, respectively. Under the strong field approximation, the interaction between the two ionized electrons is ignored by assuming that the two electrons are separated far away from each other in the final state Eq. (5). The possible effects of Coulomb interaction of the two electron in the final states may reduce the density distribution in the low momentum region on the momentum spectra. The term $\aleph_{\gamma_{1} \gamma_{2}}\left(\zeta_{i}\right)$ for $i=1,2$ in Eq. (5) is the generalized Bessel function, which can be written as [41]

$$
\begin{aligned}
\aleph_{\gamma_{1} \gamma_{2}}\left(\zeta_{i}\right)= & \sum_{\gamma_{3} \gamma_{4} \gamma_{5} \gamma_{6}} J_{-\gamma_{1}+2 \gamma_{3}+\gamma_{5}+\gamma_{6}}\left(\varsigma_{1}\right) J_{-\gamma_{2}+2 \gamma_{4}+\gamma_{5}-\gamma_{6}}\left(\varsigma_{2}\right) \\
& \times J_{-\gamma_{3}}\left(\varsigma_{3}\right) J_{-\gamma_{4}}\left(\varsigma_{4}\right) J_{-\gamma_{5}}\left(\varsigma_{5}\right) J_{-\gamma_{6}}\left(\varsigma_{6}\right),
\end{aligned}
$$

where

$$
\begin{aligned}
\varsigma_{1} & =2 \sqrt{\frac{u_{p_{1}}}{\omega_{1}}} \mathbf{p}_{i} \cdot \widehat{\epsilon}_{1}, & \varsigma_{2} & =2 \sqrt{\frac{u_{p_{2}}}{\omega_{2}}} \mathbf{p}_{i} \cdot \widehat{\epsilon}_{2}, \\
\varsigma_{3} & =\frac{1}{2} u_{p_{1}}, & \varsigma_{4} & =\frac{1}{2} u_{p_{2}}, \\
\varsigma_{5} & =2 \frac{\sqrt{u_{p_{1}} \omega_{1} u_{p_{2}} \omega_{2}}}{\omega_{1}+\omega_{2}}, & \varsigma_{6} & =2 \frac{\sqrt{u_{p_{1}} \omega_{1} u_{p_{2}} \omega_{2}}}{\omega_{1}-\omega_{2}}
\end{aligned}
$$

and $J_{m}(t)$ is the Bessel function of order $m$. In above expression, $\mathbf{p}_{i}$ is the momentum of the ionized electron, and $\widehat{\epsilon}_{1}\left(\widehat{\epsilon}_{2}\right)$ is the polarization direction of the laser's electric field with frequency $\omega_{1}\left(\omega_{2}\right)$.

In the CEI process, two sets of intermediate states of the atom-laser system are assumed. One state is that an electron is ionized in a Volkov state and the other electron is bound in the ground state of $\mathrm{He}^{+}$ion, which can be expressed as $\left|\Psi_{\mathbf{p}_{1}^{\prime} m_{1} m_{2}}\left(\mathbf{r}_{1}\right)\right\rangle \otimes\left|\Phi_{1}\left(\mathbf{r}_{2}\right)\right\rangle$ corresponding to the energy $E_{m}=\mathbf{p}_{1}^{\prime 2} / 2+\left(m_{1}+1 / 2\right) \omega_{1}+\left(m_{2}+1 / 2\right) \omega_{2}+u_{p_{1}} \omega_{1}+u_{p_{2}} \omega_{2}-I_{p_{2}}$. The other intermediate state is that one electron is ionized in an Volkov state and the other is at the first excited state of $\mathrm{He}^{+}$ion, which can be expressed as $\left|\Psi_{\mathbf{p}_{1}^{\prime \prime} l_{1} l_{2}}\left(\mathbf{r}_{1}\right)\right\rangle \otimes\left|\Phi_{2}\left(\mathbf{r}_{2}\right)\right\rangle$ corresponding to the energy $E_{l}=\mathbf{p}_{1}^{\prime \prime 2} / 2+\left(l_{1}+1 / 2\right) \omega_{1}+\left(l_{2}+1 / 2\right) \omega_{2}+u_{p_{1}} \omega_{1}+u_{p_{2}} \omega_{2}-I_{p_{12}}$ with $I_{p_{12}}$ being the ionization potential of the first excited sate of $\mathrm{He}^{+}$ion. In our calculation, we find that the NSDI probability contributed by the first excited state is much larger than that by other higher excited states. Therefore, we only consider the contribution from the first excited state of $\mathrm{He}^{+}$ion to the NSDI. $\left|\Psi_{\mathbf{p}_{1}^{\prime} m_{1} m_{2}}\left(\mathbf{r}_{1}\right)\right\rangle$ and $\left|\Psi_{\mathbf{p}_{1}^{\prime \prime} l_{1} l_{2}}\left(\mathbf{r}_{1}\right)\right\rangle$ are the well-known Volkov 
states, which can be written as [41]

$$
\begin{gathered}
\left|\Psi_{\mathbf{p}_{1} k_{1} k_{2}}\left(\mathbf{r}_{1}\right)\right\rangle=V_{e}^{-1 / 2} \sum_{j_{1}=-k_{1}, j_{2}=-k_{2}}^{\infty} \exp \left\{i\left[\mathbf{p}_{1}+\left(u_{p_{1}}-j_{1}\right) \mathbf{k}_{1}+\left(u_{p_{2}}-j_{2}\right) \mathbf{k}_{2}\right] \cdot \mathbf{r}_{1}\right\} \\
\times \aleph_{j_{1} j_{2}}\left(\zeta_{1}\right)^{*} \exp \left[-i\left(j_{1} \phi_{1}+j_{2} \phi_{2}\right)\right]\left|k_{1}+j_{1}, k_{2}+j_{2}\right\rangle .
\end{gathered}
$$

By applying the completeness relation of the intermediate states together with the initial and final states, Eq. (4) can be rewritten as

$$
\begin{aligned}
T_{\mathrm{CEI}}= & \pi^{2} \sum_{\mathbf{p}_{1}^{\prime \prime} l_{1} l_{2}}^{\infty} \sum_{\mathbf{p}_{1}^{\prime} m_{1} m_{2}}^{\infty}\left\langle\Psi_{\mathbf{p}_{1} \mathbf{p}_{2} k_{1} k_{2}}\left(\mathbf{r}_{1}, \mathbf{r}_{2}\right)\left|V_{2}\right| \Psi_{\mathbf{p}_{1}^{\prime \prime} l_{1} l_{2}}\left(\mathbf{r}_{1}\right) \Phi_{2}\left(\mathbf{r}_{2}\right)\right\rangle\left\langle\Psi_{\mathbf{p}_{1}^{\prime \prime} l_{1} l_{2}}\left(\mathbf{r}_{1}\right) \Phi_{2}\left(\mathbf{r}_{2}\right)\right. \\
& \left.\times\left|U_{12}\right| \Psi_{\mathbf{p}_{1}^{\prime} m_{1} m_{2}}\left(\mathbf{r}_{1}\right) \Phi_{1}\left(\mathbf{r}_{2}\right)\right\rangle\left\langle\Psi_{\mathbf{p}_{1}^{\prime} m_{1} m_{2}}\left(\mathbf{r}_{1}\right) \Phi_{1}\left(\mathbf{r}_{2}\right)\left|V_{1}\right| \Psi_{i n_{1} n_{2}}\left(\mathbf{r}_{1}, \mathbf{r}_{2}\right)\right\rangle \\
& \times \delta\left(E_{l}-E_{i}\right) \delta\left(E_{m}-E_{i}\right) \\
= & \pi^{2} \sum_{\mathbf{p}_{1}^{\prime \prime} l_{1} l_{2}}^{\infty} \sum_{\mathbf{p}_{1}^{\prime} m_{1} m_{2}}^{\infty} T_{\mathrm{ATI} 2} T_{\mathrm{LACE}} T_{\mathrm{ATI} 1} \delta\left(E_{l}-E_{i}\right) \delta\left(E_{m}-E_{i}\right),
\end{aligned}
$$

where

$$
\begin{aligned}
T_{\mathrm{ATI} 1} & =\left\langle\Psi_{\mathbf{p}_{1}^{\prime} m_{1} m_{2}}\left(\mathbf{r}_{1}\right) \Phi_{1}\left(\mathbf{r}_{2}\right)\left|V_{1}\right| \Psi_{i n_{1} n_{2}}\left(\mathbf{r}_{1}, \mathbf{r}_{2}\right)\right\rangle \\
& =V_{e}^{-1 / 2}\left[\left(u_{p_{1}}-q_{1}\right) \omega_{1}+\left(u_{p_{2}}-q_{2}\right) \omega_{2}\right] \aleph_{q_{1} q_{2}}\left(\zeta_{1}^{\prime}\right) \Phi_{1}\left(\mathbf{p}_{1}^{\prime}\right),
\end{aligned}
$$

with $q_{1}=n_{1}-m_{1}$ and $q_{2}=n_{2}-m_{2}$ being the photon numbers absorbed by the first electron $e_{1}$ from the first and second laser fields. The term $T_{\text {ATI1 }}$ represents the ATI process that the first electron $e_{1}$ is ionized directly from the ground state of He atom to the continuous states, which is called the ATI1 process. Here we make some approximations that the ground state $\left|\Phi_{1}\left(\mathbf{r}_{1}, \mathbf{r}_{2}\right)\right\rangle$ of an atom is a production of two hydrogen-like wave functions, and the second electron is in the ground state of $\mathrm{He}^{+}$ion when the first electron is ionized in the ATI1 process [15]. The term $T_{\mathrm{LACE}}=\left\langle\Psi_{\mathbf{p}_{1}^{\prime \prime} l_{1} l_{2}}\left(\mathbf{r}_{1}\right) \Phi_{2}\left(\mathbf{r}_{2}\right)\left|U_{12}\right| \Psi_{\mathbf{p}_{1}^{\prime} m_{1} m_{2}}\left(\mathbf{r}_{1}\right) \Phi_{1}\left(\mathbf{r}_{2}\right)\right\rangle$ represents the laser-assisted collision excitation (LACE) process that the first ionized electron $e_{1}$ collides with the bound electron $e_{2}$ and sets it to the first excited state of $\mathrm{He}^{+}$ion. The term $T_{\text {ATI2 }}$ can be rewritten as

$$
T_{\mathrm{ATI} 2}=V_{e}^{-1 / 2} \delta\left(\mathbf{p}_{1}-\mathbf{p}_{1}^{\prime \prime}\right)\left[\left(u_{p_{1}}-s_{1}\right) \omega_{1}+\left(u_{p_{2}}-s_{2}\right) \omega_{2}\right] \aleph_{s_{1} s_{2}}\left(\zeta_{2}\right) \Phi_{2}\left(\mathbf{p}_{2}\right),
$$

with $s_{1}=l_{1}-k_{1}$ and $s_{2}=l_{2}-k_{2}$ being the photon numbers absorbed by the second electron $e_{2}$ from the first and second laser fields. The term $T_{\mathrm{ATI} 2}$ represents the ATI process of the electron $e_{2}$ from the first excited state of $\mathrm{He}^{+}$ion to the continuous states, which is called the ATI2 process. By using Eqs. (5) and (8) , the transition matrix for the NSDI can be written as

$$
\begin{aligned}
T_{\mathrm{CEI}}= & -4 \pi^{3} V_{e}^{-2} \sum_{q_{1} q_{2}} \sum_{s_{1} s_{2}} p_{1}\left[\left(u_{p_{1}}-s_{1}\right) \omega_{1}+\left(u_{p_{2}}-s_{2}\right) \omega_{2}\right] \aleph_{s_{1} s_{2}}\left(\zeta_{2}\right) \Phi_{2}\left(\mathbf{p}_{2}\right) \\
& \times I^{\prime}(\mathbf{P}) \aleph_{d_{1} d_{2}}\left(\zeta_{1}-\zeta_{1}^{\prime}\right) p_{1}^{\prime}\left[\left(u_{p_{1}}-q_{1}\right) \omega_{1}+\left(u_{p_{2}}-q_{2}\right) \omega_{2}\right] \aleph_{q_{1} q_{2}}\left(\zeta_{1}^{\prime}\right) \Phi_{1}\left(\mathbf{p}_{1}^{\prime}\right),
\end{aligned}
$$


where

$$
\begin{aligned}
I^{\prime}(\mathbf{P})= & \iint d \mathbf{r}_{1} d \mathbf{r}_{2} \exp \left[-i\left(\mathbf{p}_{1}-\mathbf{p}_{1}^{\prime}\right) \cdot \mathbf{r}_{1}\right] \\
& \times \Phi_{2}^{*}\left(\mathbf{r}_{2}\right) U_{12} \Phi_{1}\left(\mathbf{r}_{2}\right) .
\end{aligned}
$$

Here, $d_{1}=\left(n_{1}-q_{1}\right)-\left(k_{1}+s_{1}\right)$ and $d_{2}=\left(n_{2}-q_{2}\right)-\left(k_{2}+s_{2}\right)$ are the photon numbers absorbed

from the first and second laser fields in the LACE process, $\mathbf{p}_{1}^{\prime}$ is the final momentum of the first ionized electron $e_{1}$ in the ATI1 process, $\Phi_{2}\left(\mathbf{p}_{2}\right)$ is the wavefunction of the first excited state of $\mathrm{He}^{+}$ion in the momentum space, and $\Phi_{1}\left(\mathbf{p}_{1}^{\prime}\right)$ is approximated by the hydrogen-like wave function with $I_{p_{1}}$ in the momentum space.

\section{NUMERICAL RESULTS}

\section{The momentum spectrum and channel analysis}

We now consider NSDI process of a helium atom exposed to the IR and XUV two-color laser fields, where the intensities of IR and XUV laser fields are chosen as $I_{1}=1.0 \times$ $10^{12} \mathrm{~W} / \mathrm{cm}^{2}$ and $I_{2}=5.0 \times 10^{13} \mathrm{~W} / \mathrm{cm}^{2}$, and the frequency of IR lase field is $\omega_{1}=1.165 \mathrm{eV}$. The polarization directions of the IR and XUV laser fields are the same, and the initial phases of both laser fields are set to zero for simplicity.

Figures 1(a)-(d) show the NSDI momentum spectra of two ionized electrons parallel to the laser polarization directions through the CEI mechanism for different frequencies of XUV laser field (a) $\omega_{2}=75 \omega_{1}$, (b) $\omega_{2}=47 \omega_{1}$, (c) $\omega_{2}=19 \omega_{1}$ and (d) $\omega_{2}=13 \omega_{1}$. For comparison, Figs. 1(e)-(h) show the momentum spectra through the CI mechanism under the same laser field conditions, where the calculation formula is shown in Ref. [16, 17]. One can see that, as the energy of XUV photon decreases, the NSDI probability of CEI mechanism is gradually higher than the corresponding probability of CI mechanism. These results show that: if the energy of XUV photon is small, the ionized electron cannot obtain enough energy by absorbing XUV photons in the ATI1 process to make the bound electron ionized directly by collision, while this bound electron can be easily pumped to the excited state of $\mathrm{He}^{+}$ion by the laser-assisted collision and then is ionized from the excited state by two-color laser fields at last. Hence the CEI mechanism makes a dominant contribution to the NSDI process when the frequency of XUV laser field is low. For example, in the case of $\omega_{2}=19 \omega_{1}$, the NSDI probability of CEI mechanism is larger than that of the CI mechanism by about five orders of magnitude. 
Figure 2 shows the NSDI momentum spectra through the CEI [(a)-(c)] and CI [(d)-(f)] mechanisms for different laser intensities $I_{1}=1.0 \times 10^{11} \mathrm{~W} / \mathrm{cm}^{2}, I_{2}=5.0 \times 10^{13} \mathrm{~W} / \mathrm{cm}^{2}$ $[(\mathrm{a}),(\mathrm{d})], I_{1}=1.0 \times 10^{12} \mathrm{~W} / \mathrm{cm}^{2}, I_{2}=5.0 \times 10^{13} \mathrm{~W} / \mathrm{cm}^{2}[(\mathrm{~b}),(\mathrm{e})]$ and $I_{1}=1.0 \times 10^{12} \mathrm{~W} / \mathrm{cm}^{2}$, $I_{2}=5.0 \times 10^{12} \mathrm{~W} / \mathrm{cm}^{2}[(\mathrm{c}),(\mathrm{f})]$, in the case of $\omega_{2}=19 \omega_{1}$. One can see that the CEI mechanism still makes a major contribution to the NSDI process for different laser intensities. Furthermore, by comparing Fig. 2(b) with Figs. 2(a) and (c), it is found that the XUV laser field determines the NSDI probability and the IR laser field plays an important role in forming the structure of the momentum spectrum, which is consistent with the situation under the CI mechanism, as shown in Fig. 2(e) and Figs. 2(d) and (f). The momentum spectra caused by CI mechanism have been investigated in the previous paper [16, 17]. Therefore, we focus on the NSDI process dominated by the CEI mechanism in this paper.

Now we take the NSDI momentum in Fig. 1(c) as example for analyzing the CEI mechanism of NSDI process. One can firstly find that the momentum distributions are the same in all the four quadrants, which is quite different from that for the case of CI mechanism as shown in Fig. 1(g). This can be easily understood as follows: in the CEI process, the first electron is ionized and it excites the second electron by collision, then the second electron is ionized by the IR and XUV laser fields from the excited state afterwards. As a result, the final momentum of the first ionized electron is completely independent to that of the second electron. Additionally, the two ionized electrons are indistinguishable, so the interference patterns hold upon the exchange of the roles of the two electrons. Therefore the CEI mechanism can lead to the same momentum distribution in the four quadrants. Moreover, one can find that the NSDI momentum spectrum can be divided into two regions consisting of the high and low ionization probabilities, where the momentum spectrum shows complex patterns. In the following, we will focus on analysis of the structure of these momentum spectrum.

Based on the previous work [16, 17, 38, 46, 47], we know that the XUV laser field has a decisive effect on the ionization process in IR and XUV two-color laser fields. Figure 3 shows the momentum spectra with the atom absorbing three (a), four (b), five (c) and six (d) XUV photons in the NSDI process. One can see that the NSDI momentum spectrum in Fig. 1(c) is attributed to the processes of the atom absorbing four and five XUV photons, as shown in Figs. 3(b) and 3(c). This is because that, the double-ionization threshold is so high that the atom has to absorb at least four XUV photons to make the NSDI process happen. 
Furthermore, the processes of the atom absorbing four and five XUV photons separately correspond to the two regions of the high and low ionization probabilities, which indicates that the ionization probability rapidly decreases with the increase of the number of the XUV photons absorbed in the NSDI process.

As we mentioned in section II, we treat the NSDI as a three-step process: the first electron is ionized in the ATI1 process, then collides with the bound electron and makes it jump from the ground state to the first excited state of $\mathrm{He}^{+}$ion in the LACE process, and finally the second electron is ionized in the ATI2 process in the IR and XUV laser fields. In all the three steps, the XUV photons may be absorbed by the electrons. Hence we now define the channel as $\left(q_{2}, d_{2}, s_{2}\right)$, where $q_{2}, d_{2}$ and $s_{2}$ are the numbers of XUV photons absorbed by electrons in the ATI1, LACE and ATI2 processes, respectively. Firstly, we consider the region of high ionization probability in the NSDI spectrum, where $q_{2}+d_{2}+s_{2}=4$. Figures 4 (a)-(c) present the channel contributions of the NSDI momentum spectra for channels $(1,2,1)(a)$, $(2,1,1)(\mathrm{b})$, and $(3,0,1)(\mathrm{c})$. The contributions of the channels $(1,0,3),(1,1,2),(2,0,2)$ and the channels with the atom absorbing zero XUV photon in the ATI (including ATI1 or ATI2) process are not shown because of their ignorable contributions to the NSDI process. From Figs. 4(a)-(c), one can clearly see that the region of the high ionization probability is almost attributed to the contributions of channels $(2,1,1)$ and $(3,0,1)$, where the contribution of channel $(2,1,1)$ is the greatest. Here we notice that, the final momentum of the first ionized electron depends on both the first ionization potential $I_{p_{1}}$ of helium and also the energy difference $\Delta E$ between the ground state and the first excited state of $\mathrm{He}^{+}$ion in the LACE process. Therefore, the first electron at least absorbs three XUV photons in the ATI1 and LACE processes to overcome these energies. Since $3 \omega_{2}-I_{p_{1}}-\Delta E \approx 0$, the final momentum of the first electron for channels $(2,1,1)$ and $(3,0,1)$ is close to zero. On the other hand, the final momentum of the second ionized electron only depends on the ionization potential $I_{p_{12}}$ of the first excited state of $\mathrm{He}^{+}$ion, so the electron absorbs one XUV photon, which is much larger than the ionization threshold, resulting in that the final momentum of the second ionized electron is much greater than zero, as shown in Figs. 廿(b) and 4(c).

Based on our theory, the NSDI caused by the CEI mechanism is due to the laser-assisted collision-excitation process; hence the direction of the first ionized electron's momentum may be changed before and after the collision. Particularly, if the angle between the ionized electron's momenta before and after the collision is smaller than $\pi / 2$, we call this collision a 
forward collision; otherwise, if the angle is larger than $\pi / 2$, we call it a backward collision.

Figures 4(d)-(f) and (g)-(i) show the contributions of forward and backward collisions for channels $(1,2,1)[(\mathrm{d})$ and $(\mathrm{g})],(2,1,1)[(\mathrm{e})$ and $(\mathrm{h})]$ and $(3,0,1)[(\mathrm{f})$ and $(\mathrm{i})]$, respectively. For channel $(1,2,1)$, where the first electron absorbs only one XUV photon in the ATI1 process, one can see that the contribution of the backward collision is comparable with that of the forward collision, while the momentum distribution of backward collision is slightly broader than that of forward collision. For channels $(2,1,1)$ and $(3,0,1)$, where the first electron carries high energy after it is ionized by absorbing two or three XUV photons in the ATI1 process, the forward collisions make a major contribution to the NSDI.

Similarly, Figures 5(a)-(e) present the channel contributions of the atom absorbing five XUV photons, and the other channels are not shown due to little contributions. Here there are only five channels making contributions to the region of the low ionization probability in the NSDI process, where the contribution of the channel $(3,1,1)$ is the biggest. Furthermore, one can find that channels $(2,2,1),(3,1,1)$ and $(4,0,1)$ illustrate similar interference patterns, while channels $(2,1,2)$ and $(3,0,2)$ show similar patterns. By comparing Figs. 4(a)-(c) with Figs. [5(a)-(e), one can find that these interference patterns depend on the XUV photons absorbed in the ATI1+LACE processes and in the ATI2 process. In order to analyze the contributions of forward and backward collisions to NSDI process, figures $5(\mathrm{f})-(\mathrm{j})$ and $(\mathrm{k})$ (o) show the NSDI momentum spectra of the forward and backward collisions. One can see that the forward collisions make major contributions to the NSDI process, especially for the channels $(3,0,2),(3,1,1)$ and $(4,0,1)$. This result further tells us that, if the ionized electron carries enough energy to excite the bound electron, the forward collision dominates the NSDI process.

In order to further understand the above observation, we define subchannel as $\left(q_{2} \mid q_{1}, d_{2}, s_{2}\right)$ within the channel $\left(q_{2}, d_{2}, s_{2}\right)$, where $q_{1}$ is the number of the IR photons that the first electron absorbs $\left(q_{1}>0\right)$ or emits $\left(q_{1}<0\right)$ in the ATI1 process. Now the LACE can be treated as the process that the ionized electron with a certain value of $p_{1}^{\prime}$ collides with the parent ion, where $p_{1}^{\prime}=\sqrt{2\left[q_{2} \omega_{2}+q_{1} \omega_{1}-I_{p_{1}}-u_{p_{1}} \omega_{1}-u_{p_{2}} \omega_{2}\right]}$. In the following, we will make a detailed discussion about the contributions of forward and backward collisions for different values of $p_{1}^{\prime}$.

Taking the subchannels of $(2 \mid-2,1,1),(2 \mid 0,1,1),(2 \mid 2,1,1)$ and $(2 \mid 5,1,1)$ within the channel $(2,1,1)$ as examples, we present the NSDI momentum spectra of these subchannels in 
Figs. 6(a)-(d). One can see that the momentum of one of two ionized electrons increases with the increase of IR photons absorbed by an electron in the ATI1 process. Furthermore, we present subchannel contributions of the forward collision in Figs. 6(e)-(h) and the backward collision in Figs. 6(i)-(l) for $(2 \mid-2,1,1)[(\mathrm{e})$ and (i)], $(2 \mid 0,1,1)[(\mathrm{f})$ and (j)], $(2 \mid 2,1,1)[(\mathrm{g})$ and $(\mathrm{k})]$ and $(2 \mid 5,1,1)[(\mathrm{h})$ and $(\mathrm{l})]$. From Fig. 6, one can see that the forward collision provides a major contribution to the NSDI, while the backward collision provides a broad momentum distribution with a lower probability to the NSDI. This result indicates that the electrons absorb more IR photons in the backward collision than that in the forward collision, since the more IR photons the electron absorbs, the lower NSDI probability is and the larger value that the momentum presents.

Similarly, taking the subchannels of $(3 \mid-5,1,1),(3 \mid-2,1,1),(3 \mid 1,1,1)$ and $(3 \mid 5,1,1)$ within the channel $(3,1,1)$ as examples, we present the NSDI momentum spectra of these subchannels in Figs. 7(a)-(d) and the subchannel contributions of the forward collision in Figs. 7(e)(h), while the backward collisions are not shown because of the little contribution to the corresponding subchannel. From Figs. 7(a) to 7(d), it is found that the momentum of the first ionized electron increases with the increase of the number of the IR photons that it absorbs in the ATI1 process. In Fig. 7, one can see that the forward collision dominates the contribution to the NSDI. Furthermore, by comparing Fig. 5(d) with Figs. 7(a)-(d), one can find that the interference pattern in Fig. 5(d) is attributed to the coherent summation of these subchannel contributions. Moreover, comparing Fig. 7 with Fig. 6, it is found that, for a given $q_{1}$, the relative contribution of forward collision to backward collision for $\left(3 \mid q_{1}, 1,1\right)$ is much larger than that for $\left(2 \mid q_{1}, 1,1\right)$. This indicates the relative contribution of forward to backward collisions depends largely on the energy carried by the first ionized electron after the ATI1 process.

\section{The saddle-point approximation and energy conservation}

We now further explain why some channels illustrate similar interference patterns, and why the relative contribution of forward to backward collisions varies with the energy carried by the ionized electron in the ATI1 process, as shown in Figs. 445. We employ the saddlepoint approximation to analyze the NSDI process. As mentioned above, the NSDI can be treated as a three-step process. Firstly, we will consider the first-step process, i.e., the first electron is ionized in the ATI1 process. We may analyze this process through the Bessel 
function $\aleph_{q_{1} q_{2}}\left(\zeta_{1}^{\prime}\right)$ in Eq. (12). In our calculation, the Bessel function can be simplified as [46]

$$
\aleph_{q_{1} q_{2}}\left(\zeta_{1}^{\prime}\right) \approx J_{-q_{1}}\left(\zeta_{11}^{\prime}, \zeta_{13}^{\prime}\right) J_{-q_{2}}\left(\zeta_{12}^{\prime}\right)
$$

where

$$
J_{-q_{1}}\left(\zeta_{11}^{\prime}, \zeta_{13}^{\prime}\right)=\sum_{q_{3}} J_{-q_{1}+2 q_{3}}\left(\zeta_{11}^{\prime}\right) J_{-q_{3}}\left(\zeta_{13}^{\prime}\right)
$$

and

$$
\begin{aligned}
& \zeta_{11}^{\prime}=2 \sqrt{\frac{u_{p_{1}}}{\omega_{1}}} \mathrm{p}_{1}^{\prime} \cos \theta_{1}^{\prime}, \\
& \zeta_{12}^{\prime}=2 \sqrt{\frac{u_{p_{2}}}{\omega_{2}}} \mathrm{p}_{1}^{\prime} \cos \theta_{1}^{\prime}, \\
& \zeta_{13}^{\prime}=\frac{1}{2} u_{p_{1}},
\end{aligned}
$$

with $\theta_{1}^{\prime}$ being the angle between the momentum of the first ionized electron and polarization directions of the two laser fields in the ATI1 process.

For a certain $q_{2}$, the Bessel function $J_{-q_{1}}\left(\zeta_{11}^{\prime}, \zeta_{13}^{\prime}\right)$ in Eq. (14) can be written as [42, 48]

$$
J_{-q_{1}}\left(\zeta_{11}^{\prime}, \zeta_{13}^{\prime}\right)=\frac{1}{T_{1}} \int_{-T_{1} / 2}^{T_{1} / 2} d t \exp \left\{i\left[\zeta_{11}^{\prime} \sin \left(\omega_{1} t\right)+\zeta_{13}^{\prime} \sin \left(2 \omega_{1} t\right)+q_{1} \omega_{1} t\right]\right\}
$$

where $T_{1}=2 \pi / \omega_{1}$. On the other hand, the classical action of an electron is [17]

$$
\begin{aligned}
S_{c l}(t, \mathbf{p}) & =\frac{1}{2} \int_{0}^{t} d t^{\prime}\left[\mathbf{p}+\mathbf{A}_{c l}\left(t^{\prime}\right)\right]^{2} \\
& =\left(\frac{1}{2} \mathbf{p}^{2}+U_{p_{1}}\right) t+2 \sqrt{\frac{u_{p_{1}}}{\omega_{1}}} \sin \left(\omega_{1} t\right) \mathbf{p} \cdot \widehat{\epsilon}_{1}+\frac{1}{2} u_{p_{1}} \sin \left(2 \omega_{1} t\right),
\end{aligned}
$$

where $U_{p_{1}}$ is the ponderomotive energy from the IR laser field with the vector potential $\mathbf{A}_{c l}(t)$. By using Eq. (18) and the energy conservation $E_{i}=E_{m}$, Eq. (17) becomes

$$
J_{-q_{1}}\left(\zeta_{11}^{\prime}, \zeta_{13}^{\prime}\right)=\frac{1}{T_{1}} \int_{-T_{1} / 2}^{T_{1} / 2} d t e^{i\left[f_{\mathrm{ATI} 1}(t)\right]}
$$

where $f_{\text {ATI1 }}(t)=S_{c l}\left(t, \mathbf{p}_{1}^{\prime}\right)-\left(q_{2} \omega_{2}-I_{p_{1}}\right) t$. By applying the saddle-point approximation, the saddle-point $t_{1}^{\prime}$ satisfies $\left.f_{\mathrm{ATI} 1}^{\prime}(t)\right|_{t=t_{1}^{\prime}}=0$, leading to the energy conservation relationship in the ATI1 process

$$
\frac{\left[\mathbf{p}_{1}^{\prime}+\mathbf{A}_{c l}\left(t_{1}^{\prime}\right)\right]^{2}}{2}=q_{2} \omega_{2}-I_{p_{1}}
$$

where $q_{2}$ is the number of XUV photons absorbed by the first ionized electron in the ATI1 process. 
Secondly, we analyze the LACE process based on the properties of the Bessel function $\aleph_{d_{1} d_{2}}\left(\zeta_{1}-\zeta_{1}^{\prime}\right)$ in Eq. (12). Under the calculation condition that we use in this paper, this Bessel function can be written as

$$
\aleph_{d_{1} d_{2}}\left(\zeta_{1}-\zeta_{1}^{\prime}\right)=J_{-d_{1}}\left(\zeta_{11}-\zeta_{11}^{\prime}\right) J_{-d_{2}}\left(\zeta_{12}-\zeta_{12}^{\prime}\right)
$$

where

$$
\begin{aligned}
& \zeta_{11}=2 \sqrt{\frac{u_{p_{1}}}{\omega_{1}}} \mathrm{p}_{1} \cos \theta_{1}, \\
& \zeta_{12}=2 \sqrt{\frac{u_{p_{2}}}{\omega_{2}}} \mathrm{p}_{1} \cos \theta_{1} .
\end{aligned}
$$

Here, $\theta_{1}$ is set to zero for the final momentum of the first ionized electron along the polarization direction of the two laser fields.

The Bessel function $J_{-d_{1}}\left(\zeta_{11}-\zeta_{11}^{\prime}\right)$ in Eq. (21) can also be expressed as an integral form [42, 48]

$$
J_{-d_{1}}\left(\zeta_{11}-\zeta_{11}^{\prime}\right)=\frac{1}{T_{1}} \int_{-T_{1} / 2}^{T_{1} / 2} d t \exp \left\{i\left[\left(\zeta_{11}-\zeta_{11}^{\prime}\right) \sin \left(\omega_{1} t\right)+d_{1} \omega_{1} t\right]\right\} .
$$

By using Eq. (18) and the energy conservation $E_{m}=E_{l}$ in the LACE process, Eq. (23) can be rewritten as

$$
J_{-d_{1}}\left(\zeta_{11}-\zeta_{11}^{\prime}\right)=\frac{1}{T_{1}} \int_{-T_{1} / 2}^{T_{1} / 2} d t e^{i\left[f_{\mathrm{LACE}}(t)\right]},
$$

where $f_{\mathrm{LACE}}(t)=\Delta S_{c l}-\left(d_{2} \omega_{2}-\Delta E\right) t$ with $\Delta S_{c l}=S_{c l}\left(t, \mathbf{p}_{1}\right)-S_{c l}\left(t, \mathbf{p}_{1}^{\prime}\right)$ and $\Delta E=I_{p_{2}}-I_{p_{12}}$. Under the saddle-point approximation, this Bessel function will further become

$$
\begin{aligned}
J_{-d_{1}}\left(\zeta_{11}-\zeta_{11}^{\prime}\right) & \approx \sum_{t_{1}} \frac{1}{\sqrt{\pi}\left[d_{1}^{2}-\left(\zeta_{11}-\zeta_{11}^{\prime}\right)^{2}\right]^{1 / 4}} \\
& \times \exp \left\{-i\left[\left(\zeta_{11}^{\prime}-\zeta_{11}\right) \sin \left(\omega_{1} t_{1}\right)-d_{1} \omega_{1} t_{1}\right]\right\}
\end{aligned}
$$

where the saddle-point $t_{1}$ satisfies

$$
\frac{1}{2}\left[\mathbf{p}_{1}+\mathbf{A}_{c l}\left(t_{1}\right)\right]^{2}=\frac{1}{2}\left[\mathbf{p}_{1}^{\prime}+\mathbf{A}_{c l}\left(t_{1}\right)\right]^{2}+\left(d_{2} \omega_{2}-\Delta E\right),
$$

which is the energy conservation equation of the first ionized electron in the LACE process. It shows that the collision is an inelastic process with absorbing $d_{2}$ XUV photons and overcoming the energy $\Delta E$. Then, we obtain the following equation according to Eq. (20) and Eq. (26)

$$
\frac{1}{2}\left[\mathbf{p}_{1}+\mathbf{A}_{c l}\left(t_{1}\right)\right]^{2}=\left(q_{2}+d_{2}\right) \omega_{2}-I_{p}^{\prime}
$$


where $I_{p}^{\prime}=I_{p_{1}}+I_{p_{2}}-I_{p_{12}}$. This equation indicates that the final momentum spectrum of the first electron depends on the total XUV photons $\left(q_{2}+d_{2}\right)$ that it absorbs in the ATI1 and LACE processes. This is the reason why the structures of the momentum spectra of the subchannels $(2,1,1)$ and $(3,0,1)$ are the same.

Lastly, we will consider the third-step process, i.e., the second electron is ionized from the first excited state of $\mathrm{He}^{+}$ion by the IR+XUV two-color laser fields. Similarly, by analyzing the Bessel function $\aleph_{s_{1} s_{2}}\left(\zeta_{2}\right)$ in Eq. (12) under the saddle-point approximation, we can also obtain the energy conservation equation satisfied by the second electron in the ATI2 process

$$
\frac{\left[\mathbf{p}_{2}+\mathbf{A}_{c l}\left(t_{2}\right)\right]^{2}}{2}=s_{2} \omega_{2}-I_{p_{12}},
$$

where $s_{2}$ is the number of XUV photons absorbed by the second ionized electron in the ATI2 process. In the above, the $t_{2}$ satisfies the equation $\left.f_{\mathrm{ATI} 2}^{\prime}(t)\right|_{t=t_{2}}=0$ with $f_{\mathrm{ATI} 2}(t)=$ $S_{c l}\left(t, \mathbf{p}_{2}\right)-\left(s_{2} \omega_{2}-I_{p_{12}}\right) t$.

To explain the reason of similar interference patterns of different channels, Fig. 8 presents comparisons of ATI spectra and NSDI spectra for channel (a) $(2,1,1)$, (b) $(3,1,1)$ and (c) $(2,1,2)$. To understand the structure, we show the ATI spectra based on Eq. (10) and Eq. (11) in Figs. $8(\mathrm{~d})$ and $8(\mathrm{e})$, respectively. In Figs. $8(\mathrm{~d})$, the $I_{p_{1}}$ is replaced by $I_{p}^{\prime}$ in Eq. (10). From Figs. 8(d) and 8(e), one can see that both the ATI spectra present steplike structures [46]. According to Eq. (27), one can find that the first and second steplike structures in Fig. 8(d) are attributed to the processes of atom absorbing three and four XUV photons under the IR laser field in the ATI1+LACE processes, respectively. Similarly, the first and second steplike structures in Fig. $8(\mathrm{e})$ are due to the first-excited electron of $\mathrm{He}^{+}$ion absorbing one and two XUV photons under the IR laser field in the ATI2 process, respectively. By mapping the ATI spectrum of Fig. 8 (d) ( Fig. 8 (e)) on the spectra of Figs. 8 (a) to 8 (c) with dash (solid) lines, one can see that the outline of interference patterns of these NSDI spectra can be well reproduced by the steplike structures in the ATI spectra, which indicates that the NSDI process caused by the CEI mechanism can be decoupled into two ATI processes of the ATI1+LACE and ATI2. It is the reason why Figs. 廿(b) and प(c) illustrate the similar interference patterns, as well as Figs. 5(b), 馬(d) and 5(e) show the similar patterns, and also Figs. 5(a) and 5(c) exhibit same patterns.

On the other hand, in order to further understand the contributions of forward and backward collisions to the NSDI, as shown in Figs. 445, we focus on the LACE process by 
analyzing the Bessel function $\aleph_{d_{1} d_{2}}\left(\zeta_{1}-\zeta_{1}^{\prime}\right)$ in Eq. (12). One can find that the Bessel function determines the probability of the LACE process and can also be expressed as $\aleph_{d_{1} d_{2}}\left(\zeta_{1}-\zeta_{1}^{\prime}\right) \propto$ $J_{-d_{1}}\left(\zeta_{11}-\zeta_{11}^{\prime}\right)$ for the weighting factor $J_{-d_{2}}\left(\zeta_{12}-\zeta_{12}^{\prime}\right)$. Furthermore, the Bessel function $J_{-d_{1}}\left(\zeta_{11}-\zeta_{11}^{\prime}\right)$ is proportional to $\frac{1}{f^{1 / 4}}$, where $f=d_{1}^{2}-\frac{4 u_{p_{1}}}{\omega_{1}}\left(\mathrm{p}_{1}^{2}+\mathrm{p}_{1}^{\prime 2} \cos ^{2} \theta_{1}^{\prime}-2 \mathrm{p}_{1} \mathrm{p}_{1}^{\prime} \cos \theta_{1}^{\prime}\right)$. Hence, the smaller the value of $f$ is, the larger the value that $J_{-d_{1}}\left(\zeta_{11}-\zeta_{11}^{\prime}\right)$ can have. If $f$ infinitely goes closer to zero and $d_{1} \geq 0$, we get

$$
d_{1}=2 \sqrt{\frac{u_{p_{1}}}{\omega_{1}}\left(\mathrm{p}_{1}^{2}+\mathrm{p}_{1}^{\prime 2} \cos ^{2} \theta_{1}^{\prime}-2 \mathrm{p}_{1} \mathrm{p}_{1}^{\prime} \cos \theta_{1}^{\prime}\right)} .
$$

In the above, one can see that, for the maximum value of $J_{-d_{1}}\left(\zeta_{11}-\zeta_{11}^{\prime}\right), d_{1}$ is determined by $p_{1}$ and $\theta_{1}^{\prime}$ for a given value $p_{1}^{\prime}$.

In the LACE process, the IR laser field may be treated as a classical field in the saddlepoint approximation. Figure 9 shows IR photon numbers $d_{1}$ in the LACE process as a function of $p_{1}$, which is the momentum value of the ionized electron after collision, and $\theta_{1}^{\prime}$ with the momentum value before collision $p_{1}^{\prime}$ being (a) 0.26 a.u., (b) 1.21 a.u. and (c) 2.17 a.u., where the region of $\theta_{1}^{\prime}<\pi / 2$ in each graph represents forward collision and the region of $\theta_{1}^{\prime}>\pi / 2$ represents backward collision. One can see that, for backward collision, the number of the IR photons absorbed by the first electron increases as the value of $p_{1}^{\prime}$ increases. We have noticed that, although the atom absorbs a few IR photons (less than 12), the total energy of these IR photons is not enough to excite the bound electron, hence the source of the energy $\Delta E$ to excite the bound electron comes not only from the energy transfer between the two electrons and absorbing IR photons, but also from absorbing XUV photons during the collision. This result is consistent with the calculated result of $\mathrm{Hu}$ [38]. Furthermore, for the case of absorbing certain number of XUV photons, the probability of the LACE process is determined by the number of IR photons that electron absorbs. From Fig. 9, it is found that: (i) when the value of $p_{1}^{\prime}$ is small, the numbers of the IR photons absorbed in both the forward and backward collisions are about equal as shown in Fig. 9 (a), and hence the forward and backward collisions make a comparable contribution to the NSDI, as shown in Figs. 4(d) and 4(g); (ii) the number of the IR photons absorbed in the forward collision is less than that in the backward collision when the value of $p_{1}^{\prime}$ becomes larger as shown in Fig. 9 (b), which indicates that the forward collision makes a major contribution to the NSDI, while the backward collision provides a broader momentum distribution, as shown in Fig. 6, (iii) when the ionized electron carries enough energy to excite the bound 
electron by collision without absorbing the XUV photons in the LACE process, the electron absorbs much less IR photons in the forward collision than that it does in backward collision as shown in Fig. 9 (c), thus the forward collision plays a dominant role in the NSDI, as shown in Fig. 4(f) as well as Figs. 5(c)and (e). To summarize the above analysis, with increase of the value of $p_{1}^{\prime}$, the source of the energy $\Delta E$ to excite the bound electron changes from absorbing XUV photons to the pure energy transfer between the two electrons in the LACE process, and hence the forward collision gradually becomes dominant in the NSDI, as shown in Figs. 4, 7 ,

In contrast to the above results, the backward collision makes a dominant contribution to the NSDI in the case of a monochromatic IR laser field, which is shown in Fig.8 of Ref. [15]. Here we may notice that, in a monochromatic IR laser field, the amplitude of the LACE process only depends on Eq. (23), and hence the small value of $p_{1}^{\prime}$ in the ATI1 process makes a crucial contribution to the NSDI. In order to explain the difference, Fig. 10 shows a comparison of IR photon number $d_{1}$ in the LACE process under the monochromatic IR (a) and IR+XUV two-color (b) laser fields for $p_{1}^{\prime}=0.26$ a.u.. The intensity and frequency of monochromatic IR laser field in Fig. 10(a) are $I=2.2 \times 10^{14} \mathrm{~W} / \mathrm{cm}^{2}$ and $\omega=1.165 \mathrm{eV}$. Figure 10(b) shows a part of Fig. 9(a) for small value of $p_{1}$. One can see that, the electron absorbs more IR photons for the backward collision than that for the forward collision in both cases, while the range of the number of IR photons absorbed in the monochromatic IR laser field is much larger than that in two-color laser fields. Furthermore, in Fig. 10(a), one can find that the energy obtained in the forward collision is so little that the forward collision can not make the bound electron to be excited. On the contrary, the electron can absorb lots of IR photons in the backward collision, and hence the bound electron may have a chance to be excited in the LACE process. As a result, the backward collision makes a crucial contribution to the NSDI in the monochromatic IR laser field.

\section{CONCLUSIONS}

Based on the frequency-domain theory, we investigate the CEI mechanism of NSDI process for a helium atom in the IR+XUV two-color laser fields. We find that the NSDI probability of the CEI mechanism is much larger than that of the CI mechanism when the

XUV photon energy is smaller than the ionization threshold of $\mathrm{He}^{+}$ion. It shows that the 
NSDI momentum spectrum caused by the CEI mechanism presents complex interference

patterns and symmetrical structures. With the help of channel analysis, we find that the momentum spectrum is attributed to the interference between different channels. With the channel number increases, the energy of the ionized electron increases in the ATI1 process, and hence the source of the energy to excite the bound electron changes from absorbing XUV photons to pure energy transfer between two electrons during the LACE process. Furthermore, in order to explain the interference pattern, we investigated the contributions to the NSDI from the forward and backward collisions. We found that, if the energy of recolliding electron is small, both the forward and backward collisions play comparable roles in the NSDI, while the distribution of momentum spectrum is broader for the backward collision than for the forward; on the other side, if the the energy of recolliding electron is very large, the forward collision plays a dominant role in the NSDI. Also, we employed the saddle-point approximation to obtain the equation of energy conservation, and reconstructed the formation of interference patterns of different channels by the spectra of two ATI processes. In addition, we also explained the reason why the backward collision plays a dominant role in the CEI mechanism of NSDI under the monochromatic IR laser field.

\section{ACKNOWLEDGMENTS}

This research was supported by the National Natural Science Foundation of China under Grant Nos. 11474348, 61275128, 11334009 and 11425414 . C. J. was supported by the National Basic Research Program of China Grant No. 2013CB922201. Z.-C.Y. was supported by the Natural Sciences and Engineering Research Council of Canada and by the Canadian computing facilities of SHARCnet, and ACEnet.

[1] C. Figueira de Morisson Faria, and X. Liu, J. Mod. Opt. 58, 1076 (2011); W. Becker, X. Liu, P. J. Ho, and J. H. Eberly, Rev. Mod. Phys. 84, 1011 (2012).

[2] A. l'Huillier, L. A. Lompre, G. Mainfray, and C. Manus, Phys. Rev. A 27, 2503 (1983).

[3] P. B. Corkum, Phys. Rev. Lett. 71, 1994 (1993).

[4] J. S. Parker, B. J. S. Doherty, K. T. Taylor, K. D. Schultz, C. I. Blaga and L. F. DiMauro, Phys. Rev. Lett. 96, 133001 (2006). 
[5] S. P. Goreslavski and S. V. Popruzhenko, Opt. Express 8, 395 (2001).

[6] S. P. Goreslavskii, S. V. Popruzhenko, R. Kopold, and W. Becker, Phys. Rev. A 64, 053402 (2001).

[7] R. Kopold, W. Becker, H. Rottke, and W. Sandner, Phys. Rev. Lett. 85, 3781 (2000).

[8] C. Figueira de Morisson Faria and M. Lewenstein, J. Phys. B: At. Mol. Opt. Phys. 38, 3251 (2005)

[9] X. Hao, J. Chen, W. Li, B. Wang, X. Wang and W. Becker, Phys. Rev. Lett. 112, 073002 (2014).

[10] H. Li, J. Chen, H. Jiang, J. Liu, P. Fu, Q. Gong, Z.-C. Yan, and B. Wang J. Phys. B: At. Mol. Opt. Phys. 42, 125601 (2009).

[11] X. L. Hao, G. Q. Wang, X. Y. Jia, W. D. Li, J. Liu, and J. Chen, Phys. Rev. A 80, 023408 (2009); J. Chen, J. Liu, L. B. Fu, and W. M. Zheng, ibid. 63, 011404(R) (2000).

[12] D. F. Ye, X. Liu, and J. Liu, Phys. Rev. Lett. 101, 233003 (2008).

[13] P. J. Ho, R. Panfili, S. L. Haan, and J. H. Eberly, Phys. Rev. Lett. 94, 093002 (2005); P. J. Ho and J. H. Eberly, ibid. 97, 083001 (2006).

[14] F. Mauger, C. Chandre, and T. Uzer, Phys. Rev. Lett. 105, 083002 (2010).

[15] B. Wang, Y. Guo, J. Chen, Z.-C. Yan and P. Fu, Phys. Rev. A 85, 023402 (2012).

[16] F. Jin, Y. Tian, J. Chen, Y. Yang, X. Liu, Z.-C. Yan and B. Wang, Phys. Rev. A 93, 043417 (2016).

[17] F. Jin, J. Chen, Y. Yang, Z.-C. Yan and B. Wang, J. Phys. B: At. Mol. Opt. Phys. 49, 195602 (2016).

[18] B. Bergues, M. Kübel, N. G. Johnson et al., Nat. Commun. 3, 813 (2012).

[19] C. Huang, Y. Zhou, Q. Zhang and P. Lu, Opt. Express 21, 11382 (2013).

[20] Z. Zhang, J. Zhang, L. Bai and X. Wang, Opt. Express 23, 7044 (2015).

[21] T. Shaaran, M. T. Nygren and C. Figueira de Morisson Faria, Phys. Rev. A 81, 063413 (2010);

C. Figueira de Morisson Faria, T. Shaaran and M. T. Nygren, ibid. 86, 053405 (2012); A. S. Maxwell and C. Figueira de Morisson Faria, ibid. 92, 023421 (2015).

[22] A. S. Maxwell and C. Figueira de Morisson Faria, Phys. Rev. Lett. 116, 143001 (2016).

[23] S. Dong, X. Chen, J. Zhang and X. Ren, Phys. Rev. A 93, 053410 (2016).

[24] Z. Yuan, D. Ye, J. Liu and L. Fu, Phys. Rev. A 93, 063409 (2016).

[25] X. Sun, M. Li, D. Ye, G. Xin, L. Fu, X. Xie, Y. Deng, C. Wu, J. Liu, Q. Gong and Y. Liu, 
Phys. Rev. Lett. 113, 103001 (2014).

[26] S. Eckart, et al., Phys. Rev. Lett. 117, 133202 (2016).

[27] T. Shintake et al., Nat. Photonics 2, 555 (2008).

[28] P. Emma, R. Akre, et al., Nat. Photonics 4, 641 (2010).

[29] S. Rosenblum, O. Bechler, I. Shomroni, Y. Lovsky, G. Guendelman and B. Dayan, Nat. Photonics 10, 19 (2016).

[30] T. Popmintchev, M.-C. Chen, P. Arpin, M. M. Murnane and H. C. Kapteyn, Nat. Photonics 4, $822(2010)$.

[31] M. Chini, K. Zhao and Z. Chang, Nat. Photonics 8, 178 (2014).

[32] Y. Pan, S.-F. Zhao, and X.-X. Zhou, Phys. Rev. A 87, 035805 (2013).

[33] J S Briggs and V Schmidt, J. Phys. B: At. Mol. Opt. Phys. 33, R1 (2000).

[34] L.-Y. Peng, W.-C. Jiang, J.-W. Geng, W.-H. Xiong and Q. Gong, Physics Reports 575, 1 (2015).

[35] W.-C. Jiang, J.-Y. Shan, Q. Gong and L.-Y. Peng, Phys. Rev. Lett. 115, 153002 (2015).

[36] Z. Zhang, L.-Y. Peng, M. Xu, A. F. Starace, T. Morishita and Q. Gong, Phys. Rev. A 84, 043409 (2011).

[37] A. Liu and U. Thumm, Phys. Rev. Lett. 115, 183002 (2015).

[38] S. X. Hu, Phys. Rev. Lett. 111, 123003 (2013).

[39] A. Liu and U. Thumm, Phys. Rev. A 91, 043416 (2015); ibid. 89, 063423 (2014).

[40] M. Gell-Mann and M. L. Goldberger, Phys. Rev. 91, 398 (1953); M. L. Goldberger and K. M. Watson, Collision Theory (Wiley, New York, 1964).

[41] D.-S. Guo and T. Åberg, J. Phys. A 21, 4577 (1988); D.-S. Guo, T. Åberg and B. Crasemann, Phys. Rev. A 40, 4997 (1989); D.-S. Guo and G W F Drake, J. Phys. A: Math. Gen. 25, 5377 (1992).

[42] B. Wang, L. Gao, X. Li, D.-S. Guo and P. Fu, Phys. Rev. A 75, 063419 (2007).

[43] B. Wang, Y. Guo, B. Zhang, Z. Zhao, Z.-C. Yan and P. Fu, Phys. Rev. A 82, 043402 (2010).

[44] P. Fu, B. Wang, X. Li and L. Gao, Phys. Rev. A 64, 063401 (2001).

[45] L. Gao, X. Li, P. Fu, R. R. Freeman and D.-S. Guo and P. Fu, Phys. Rev. A 61, 063407 (2000).

[46] K. Zhang, J. Chen, X.-L. Hao, P. Fu, Z.-C. Yan and B. Wang, Phys. Rev. A 88, 043435 (2013).

[47] M. Liu, Y. Guo and B. Wang, Chin. Phys. B 24, 073201 (2015). 
[48] Y. Guo, P. Fu, Z.-C. Yan, J. Gong and B. Wang, Phys. Rev. A 80, 063408 (2009). 

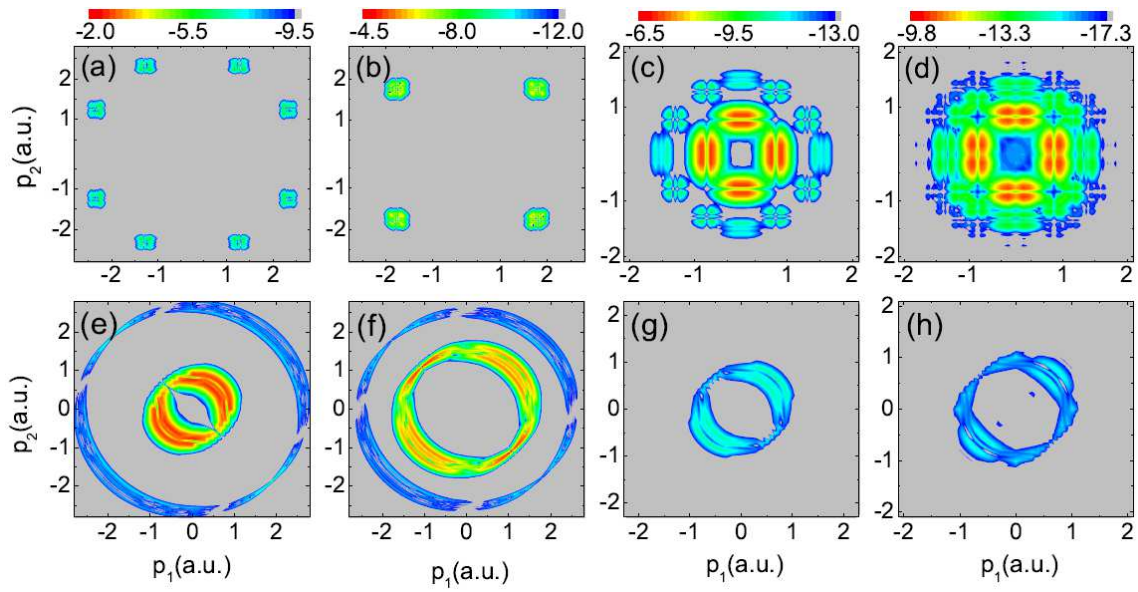

Figure 1: (Color online). Momentum spectra of two ionized electrons along the laser polarization directions through $[(\mathrm{a})-(\mathrm{d})]$ the collision-excitation ionization and $[(\mathrm{e})-(\mathrm{h})]$ the collision-ionization processes for different laser frequencies $\omega_{2}=75 \omega_{1}[(\mathrm{a}),(\mathrm{e})], \omega_{2}=47 \omega_{1}[(\mathrm{~b}),(\mathrm{f})], \omega_{2}=19 \omega_{1}[(\mathrm{c}),(\mathrm{g})]$ and $\omega_{2}=13 \omega_{1}[(\mathrm{~d}),(\mathrm{h})]$, where the intensities of IR and XUV laser fields are $I_{1}=1.0 \times 10^{12} \mathrm{~W} / \mathrm{cm}^{2}$ and $I_{2}=5.0 \times 10^{13} \mathrm{~W} / \mathrm{cm}^{2}$, and the frequency of IR lase field is $\omega_{1}=1.165 \mathrm{eV}$. In logarithmic scale.
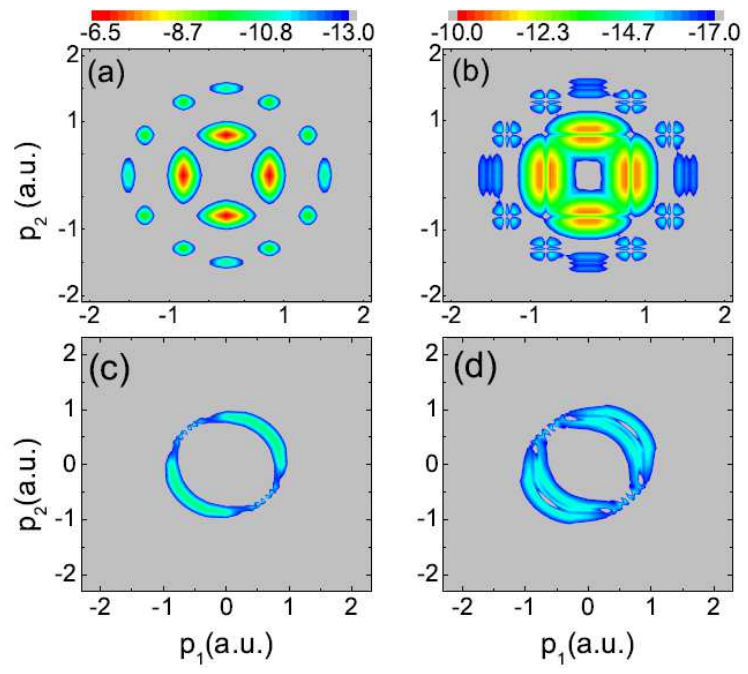

Figure 2: (Color online). The NSDI momentum spectra through the CEI [(a)-(c)] and CI [(d)(f)] mechanisms for different laser intensities $I_{1}=1.0 \times 10^{11} \mathrm{~W} / \mathrm{cm}^{2}, I_{2}=5.0 \times 10^{13} \mathrm{~W} / \mathrm{cm}^{2}$ $[(\mathrm{a}),(\mathrm{d})], I_{1}=1.0 \times 10^{12} \mathrm{~W} / \mathrm{cm}^{2}, I_{2}=5.0 \times 10^{13} \mathrm{~W} / \mathrm{cm}^{2}[(\mathrm{~b}),(\mathrm{e})]$ and $I_{1}=1.0 \times 10^{12} \mathrm{~W} / \mathrm{cm}^{2}$, $I_{2}=5.0 \times 10^{12} \mathrm{~W} / \mathrm{cm}^{2}[(\mathrm{c}),(\mathrm{f})]$, where the frequency of XUV laser field is $\omega_{2}=19 \omega_{1}$. In logarithmic scale. 

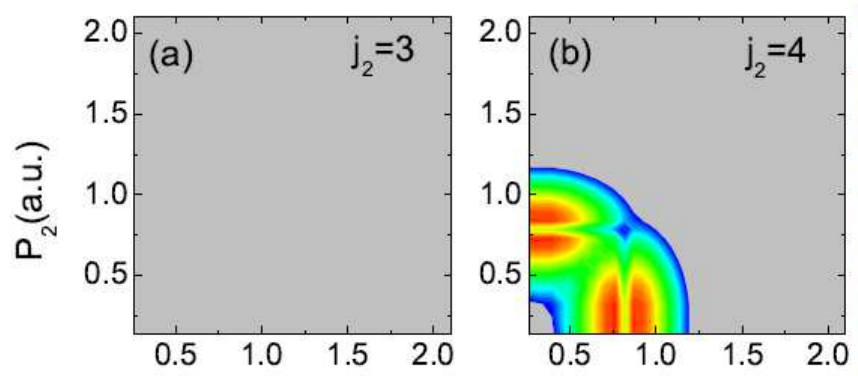

$-6.5$
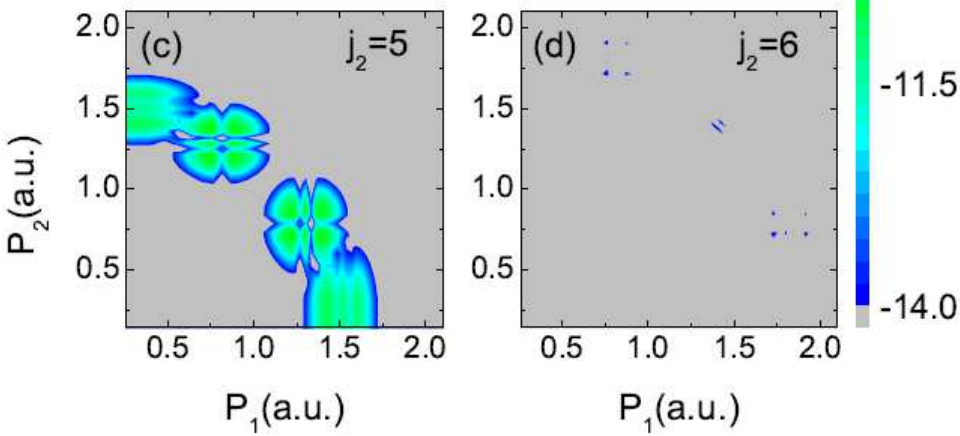

Figure 3: (Color online). Momentum spectra of the atom totally absorbing (a) $j_{2}=3$, (b) $j_{2}=4$, (c) $j_{2}=5$ and (d) $j_{2}=6$ XUV photons in the NSDI process. In logarithmic scale.
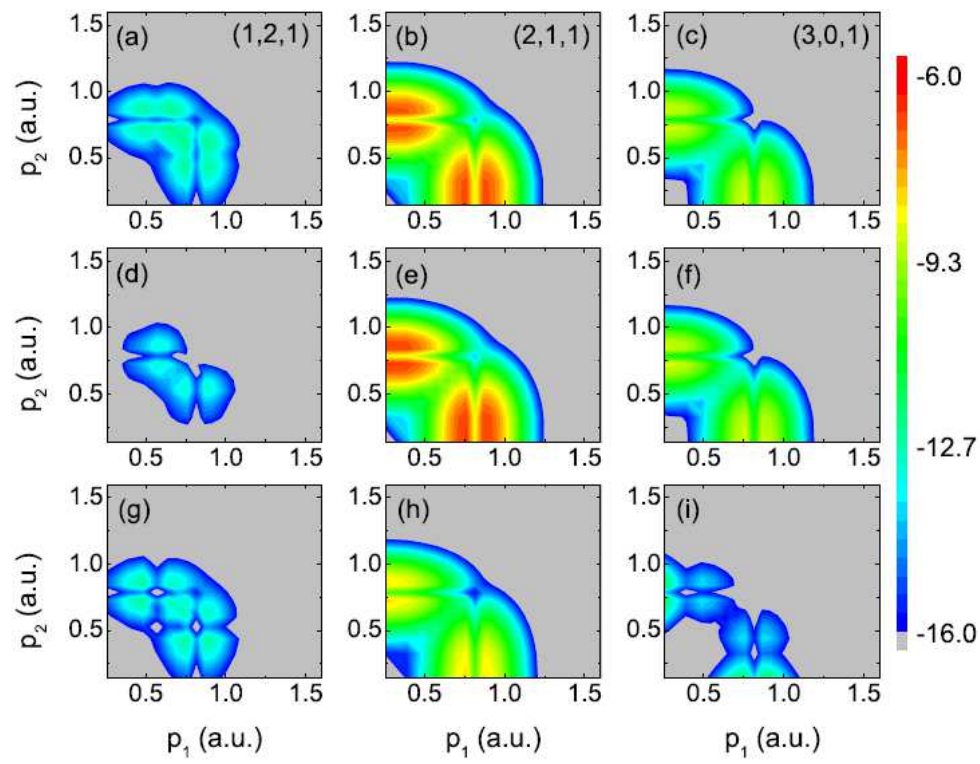

Figure 4: (Color online). Channel contributions of the NSDI momentum spectra for channels (a) $(1,2,1)$, (b) $(2,1,1),(\mathrm{c})(3,0,1)$. (d)-(f) and (d)-(f) present the contributions of forward and backward collisions for channels $[(\mathrm{d}),(\mathrm{g})](1,2,1),[(\mathrm{e}),(\mathrm{h})](2,1,1)$ and $[(\mathrm{f}),(\mathrm{i})](3,0,1)$, respectively. In logarithmic scale. 

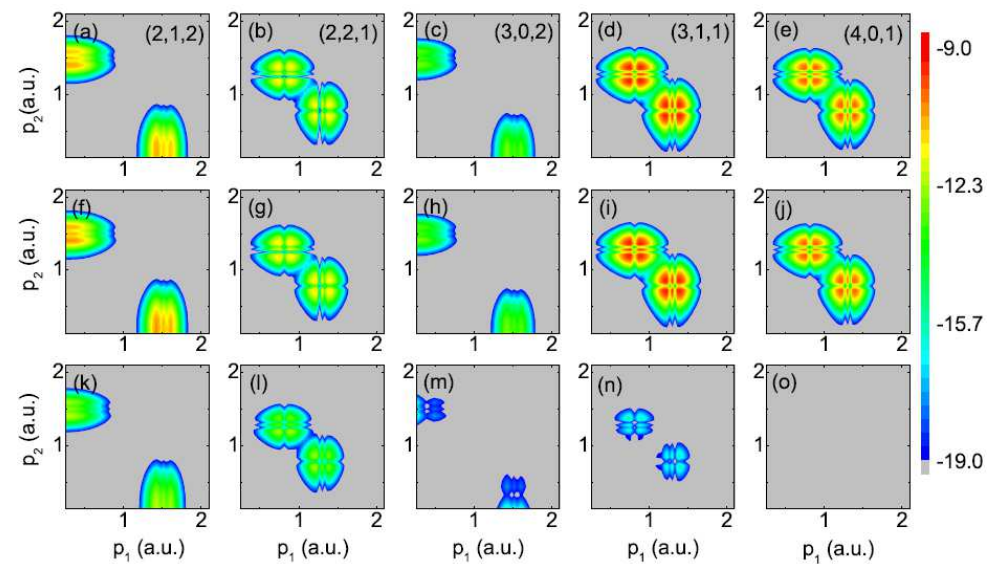

Figure 5: (Color online). Channel contributions of the NSDI momentum spectra for channels (a) $(2,1,2)$, (b) $(2,2,1),(\mathrm{c})(3,0,2),(\mathrm{d})(3,1,1)$ and (e) $(4,0,1)$. (f)-(j) and (d)-(f) present the contributions of forward and backward collisions for channels [(f), (k)] $(2,1,2),[(\mathrm{g}),(\mathrm{l})](2,2,1)$, $[(\mathrm{h}),(\mathrm{m})](3,0,2),[(\mathrm{i}),(\mathrm{n})](3,1,1)$ and $[(\mathrm{j}),(\mathrm{o})](4,0,1)$, respectively. In logarithmic scale.
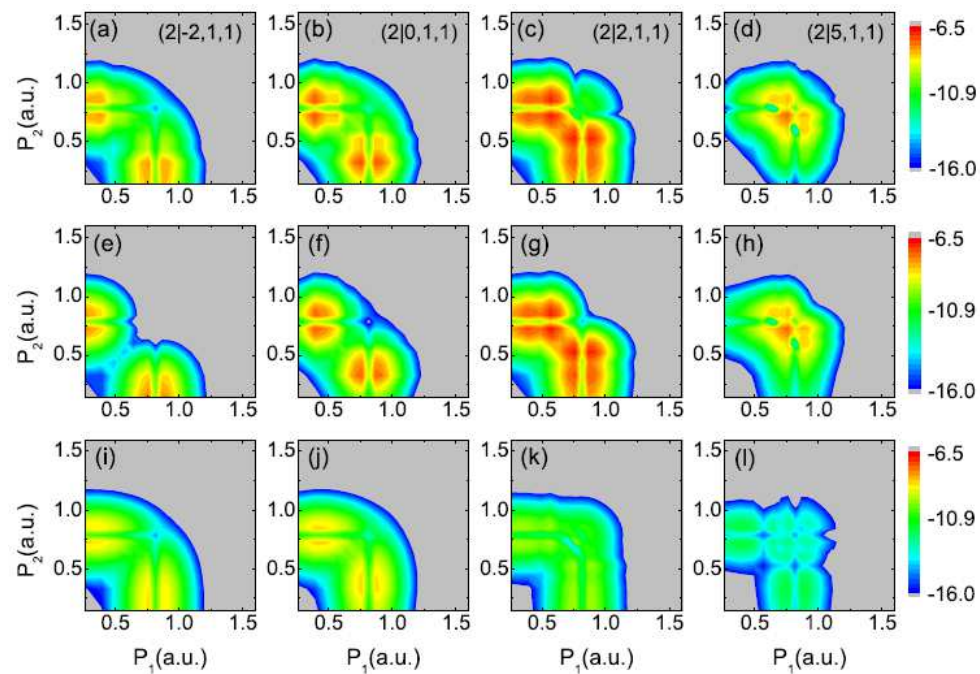

Figure 6: (Color online). Subchannel contributions of the NSDI momentum spectra for (a) (2|$2,1,1)$, (b) $(2 \mid 0,1,1)$, (c) $(2 \mid 2,1,1)$ and (d) $(2 \mid 5,1,1)$. (e)-(h) and (i)-(l) present the NSDI momentum spectra of the forward and backward collisions for subchannels [(e), (i)] (2|-2,1,1), [(f), (j)] $(2 \mid 0,1,1)$, $[(\mathrm{g}),(\mathrm{k})](2 \mid 2,1,1)$ and $[(\mathrm{h}),(\mathrm{l})](2 \mid 5,1,1)$, respectively. In logarithmic scale. 

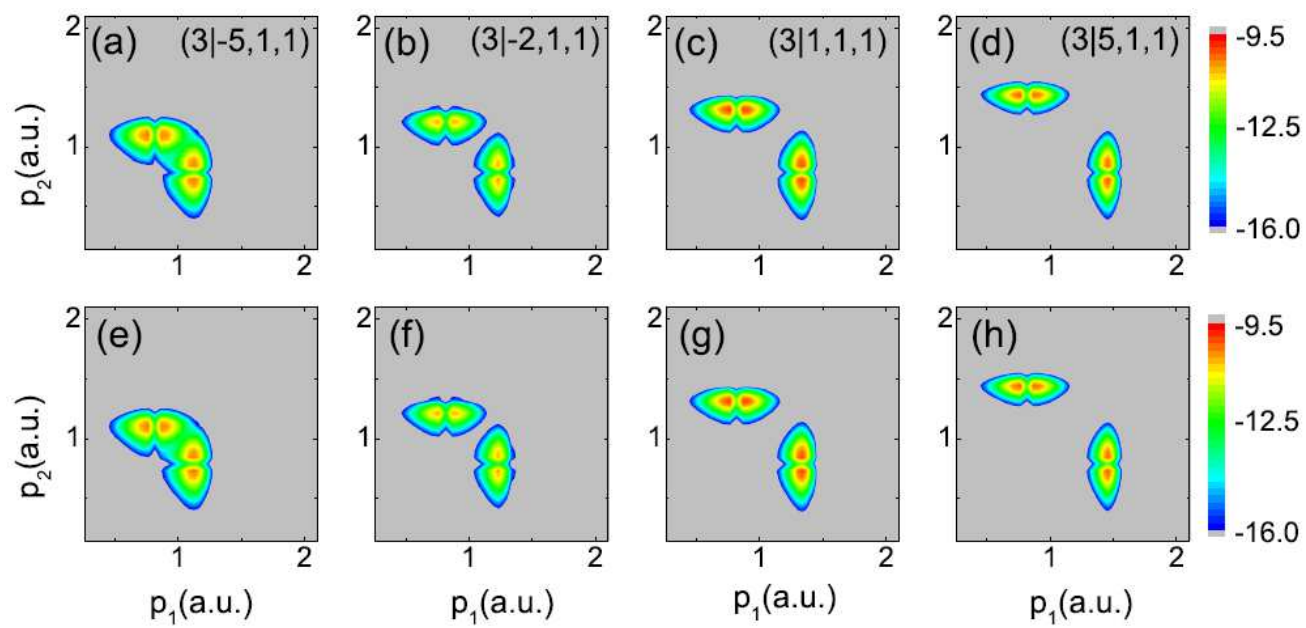

Figure 7: (Color online). Subchannel contributions of the NSDI momentum spectra for (a) (3|$5,1,1)$, (b) $(3 \mid-2,1,1)$, (c) $(3 \mid 1,1,1)$ and (d) $(3 \mid 5,1,1)$. (e)-(h) present the NSDI momentum spectra of the forward collision for subchannel (e) $(3 \mid-5,1,1)$, (f) $(3 \mid-2,1,1)$, (g) $(3 \mid 1,1,1)$ and (h) $(3 \mid 5,1,1)$. In logarithmic scale.
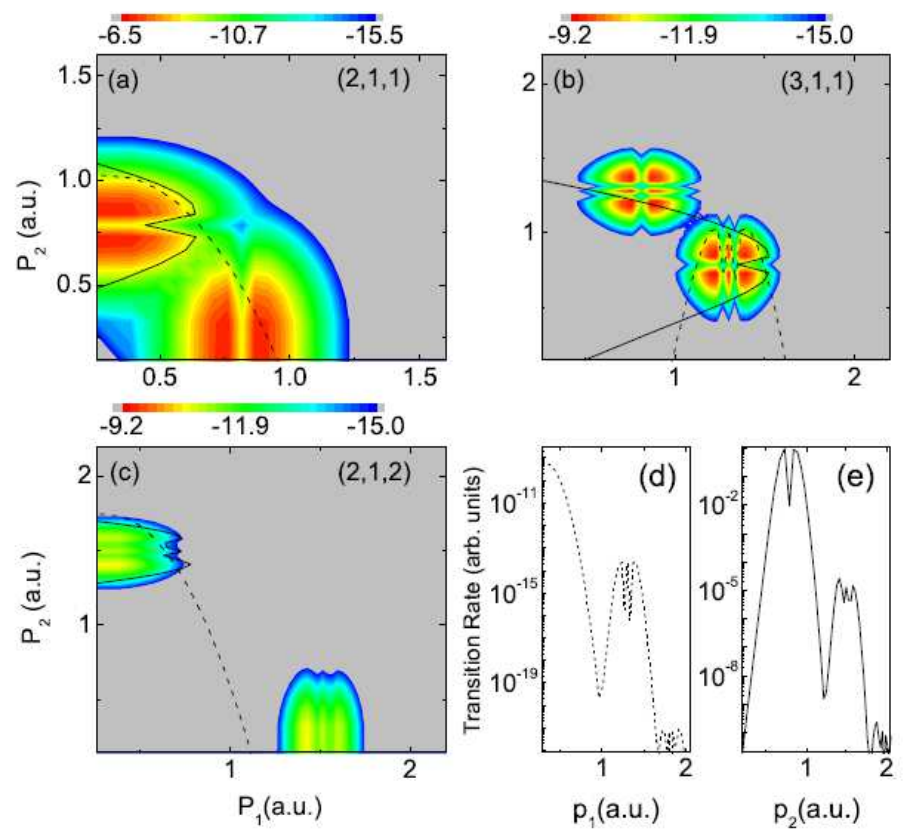

Figure 8: (Color online). Comparisons of ATI spectra and NSDI spectra for channel (a) $(2,1,1)$, (b) $(3,1,1)$ and (c) $(2,1,2)$. The dash lines in (a), (b) and (c) represent the ATI spectrum based on Eq. (10) with $I_{p_{1}}$ replaced by $I_{p}^{\prime}$, where this ATI spectrum is shown in (d); and the solid lines in (a), (b) and (c) represent the ATI spectrum based on Eq. (11), where this ATI spectrum is shown (e). 

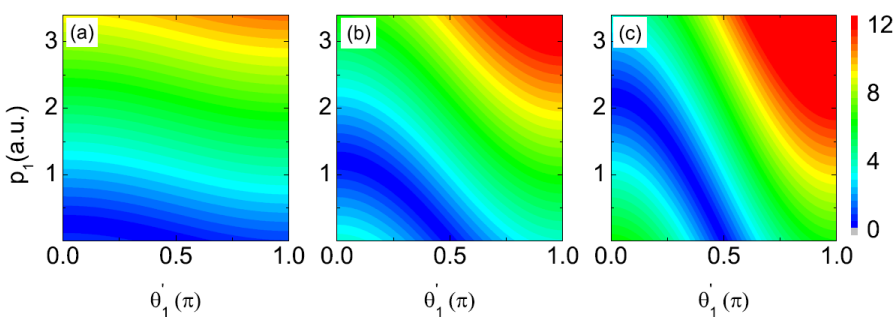

Figure 9: (Color online). Number of atoms absorbing IR photons in the LACE process for (a) $p_{1}^{\prime}=0.26$ a.u., (b) $p_{1}^{\prime}=1.21$ a.u. and (c) $p_{1}^{\prime}=2.17$ a.u., where the direction of the momentum $\mathbf{p}_{1}^{\prime}$ varies from 0 to $\pi$, and the momentum $\mathbf{p}_{1}$ is along the IR laser polarization direction. The region of $\theta_{1}^{\prime}<\pi / 2$ in each graph represents forward collision and the region of $\theta_{1}^{\prime}>\pi / 2$ in each graph represents backward collision.
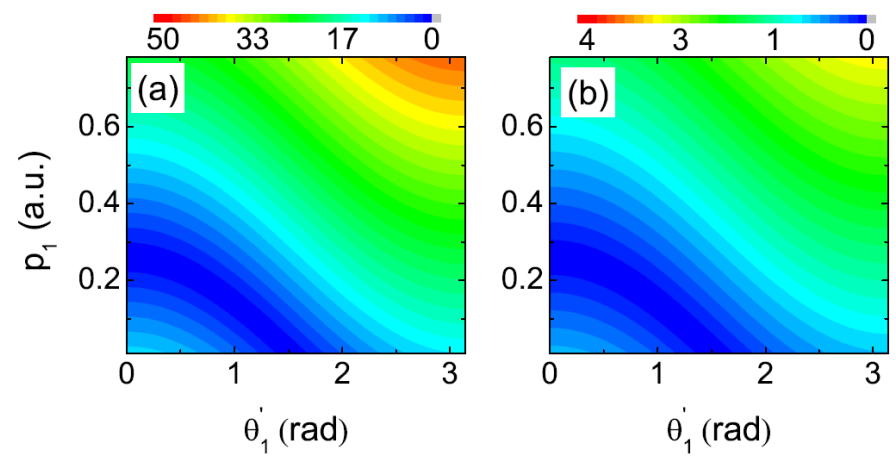

Figure 10: (Color online). Comparisons of absorbing IR photons in the LACE process under (a) the monochromatic IR and (b) IR+XUV two-color laser fields for $p_{1}^{\prime}=0.26$ a.u.. The left is for the intensity and frequency of IR laser field being $I=2.2 \times 10^{14} \mathrm{~W} / \mathrm{cm}^{2}$ and $\omega=1.165 \mathrm{eV}$, and the right is the part of Fig. 9(a) for small value $p_{1}$. The region of $\theta_{1}^{\prime}<\pi / 2$ in each graph represents forward collision and the region of $\theta_{1}^{\prime}>\pi / 2$ in each graph represents backward collision. 